\title{
DEVELOPMENT OF INDIVIDUAL HOME SECURITY SYSTEM USING CAN AND ZIGBEE PROTOCOL
}

\author{
P.Mohan $^{1}$, M. Vinoth Kumar ${ }^{2}$ \\ ${ }^{1}$ PG Scholar, Masters Degree in Embedded System Technologies, Rajiv Gandhi College of Engineering, Chennai, \\ Tamilnadu, India \\ ${ }^{2}$ Assistant professor, Department of Electrical and Electronics Engineering, Rajiv Gandhi College of Engineering, \\ Chennai, Tamilnadu, India
}

\begin{abstract}
This paper proposes a security alert system developed using CAN interface and ZigBee protocols implemented with GSM technology. Whenever the system notices human intrusion or senses leakage of raw gas, immediately sends a Theft or Sensor alert message to the authorities using GSM Modem and Passing alert messages to nearby ZigBee networked houses, and also the controller triggers the exhaust fan in the case of gas leakage. The major advantage of the system is to access the secret coded electromagnetic lock using GSM mobile that provides maximum security to the house. In the case of owner's absence in the house, the system takes over the control of all the equipments, and saves the energy by turning-off unused electrical appliances.
\end{abstract}

Keywords: Controller Area Network (CAN), ZigBee Protocol, Electromagnetic lock

\section{INTRODUCTION}

In today's fast growing environment we concern for security and convenience of daily life. Most of developed countries certainly invested more money and manpower to research and develop a highly protected home security system and have achieved great success. In this project we develop an individual home security alert system by implementing CAN, ZigBee protocols along with GSM technology. The system can detect the theft, leaking of raw gas and fire, and immediately send an alert message using GSM Modem.

The hardware of this system consists of the wired CAN module, wireless ZigBee Transceiver, as well as the GSM module. Whenever the controller detects any human movement or leakage of gas inside the house immediately sends the alert message to owner and police station via GSM network and also intimates to the nearby ZigBee nodes using ZigBee transceiver for a quick response. This system limits the electricity power usage by automatically shutting down unused electrical device when the owner not present in the house. The password protected electromagnetic lock to open and close the house using GSM technology takes the security of the house to the next level. Information sharing among the nearby wireless node provides quick response to any kind of alerts by the incident house.

Following are section in which order the project has been explained in detail,

$>$ Section[2] Related works

$>$ Section[3] proposed System
$>$ Section[4] System Requirements

$>$ Section[5] General Block Diagram and Description

$>$ Section[6] Conclusion and Future work

\section{RELATED WORKS}

The home appliances can be monitored and controlled locally using the embedded system board from a remote location through a web browser from anywhere in the world with an internet access. The system is scalable and allows multi-vendor appliances to be added, without a major hardware modification. The Internet has also started to serve as a medium that allows the monitoring, control and interaction with machine and devices from a remote place.

The Internet based home automation system that provides many features ranging from efficient use of energy to increased comfort, greater safety and security. Even over large distances the user can monitor and control his/her home gate, oven, refrigerator and the water supply to the garden without any human intervention.

Despite these attractions and benefits, home automation has not yet received broad acceptance and attention. This is mainly due to its high cost and complexity as well as the lack of security. Some of the related works for this proposed system encouraged me doing this project, the authors Khusvinder Gill, Shuang-Hua Yang, Wan-Liang Wang using internet for communication between the system and the user in his paper, a Secure Remote Access to Home Automation Networks, that makes the system costlier and give way to use new method for 
communication with low cost, with this idea, the proposed project developed with the low cost device with portability to be carried to the remote places easily.

\section{PROPOSED SYSTEM}

The proposed system introduces a wired sensor network based home security system, in which the control of the devices are completely taken over by the system, the process of monitoring and sending the alert messages become more important. The system can detect the theft, leaking of raw gas and fire, and immediately sends the alert message to owner and police station via GSM network and also intimates to the nearby ZigBee nodes using ZigBee transceiver for a quick response. This system limits the electricity power usage by automatically turning off the unused electrical device when the owner not present in the house. In addition to all these features, the password protected electromagnetic lock to open and close the house using GSM technology takes the security of the house to the next level.

The hardware of this system consist of the CAN Module, wireless sending and receiving chip ZigBee Transceiver, as well as the GSM module. Depending on the alert from sensors, the messages sent to the authorized personnel via Global System for Mobile Communications (GSM) using Short Message Service (SMS). It is a Low power-consuming and the capability of battery operated security monitoring system. The wired communication among the sensor networks reduces electromagnetic interference to human specifically for senior citizen old aged persons in houses.

\section{SYSTEM REQUIREMENTS}

\subsection{Hardware Requirements:}

$\begin{array}{lll}\text { PIC Microcontroller } & - & \text { PIC16F877A } \\ \text { CAN Controller } & - & \text { MCP 2515 } \\ \text { CAN Transceiver } & - & \text { MCP 2551 } \\ \text { LPG Gas Sensor } & - & \text { MQ-6 } \\ \text { PIR Sensor } & - & \text { SB0061 } \\ \text { Electromagnetic lock } & - & \text { 12v DC } \\ \text { Zigbee transceiver } & - & \text { XBEE S1 (802.15.4) }\end{array}$

\subsection{Software Requirements:}

MPLAB IDE v8.53

WinPIC800 Programmer

Embedded C Language

\section{GENERAL BLOCK DIAGRAM AND DESCRIPTION}

\subsection{PIC Microcontroller}

Microcontroller PIC16F877A is one of the PICMicro Family controllers, which is popular among the all professionals.
Because of FLASH memory technology that can be write-erase until thousand times. The major advantage this RISC Microcontroller compared to with other Microcontroller 8-bit especially at a speed of and his code compression technique, which only have 40 pin with 33 pins used for I/O operation.

PIC16F877A perfectly suited for various industries starting from automotive industries and controlling of home appliances to industrial instruments by monitoring remote sensors and electrical door locks, and providing safety to devices. It is ideal for smart cards as well as for battery supplied devices because of its low power utilization. EEPROM memory makes it easier to apply microcontrollers to devices where permanent storage of various parameters is needed such as the codes for transmitters, motor speed, and receiver frequencies. With the Low cost and low power consumption, the flexibility of handling the PIC16F877A controller makes applicable for replacement in larger systems and coprocessor application.

In System Programmability of this chip with using only two pins in data transfer that makes possible the flexibility of a product, even after completing the assembling and testing of the product. This potential can be used to create assembly-line production that can store calibration data available only after final testing and used to improve programs on finished products. . In our project we implement CAN communication protocol for the communication between two controllers and in addition to that in future the number of nodes can be increased without major modification in the Hardware.

\subsection{CAN Controller}

Controller Area Network (CAN) is a serial communication network that was initially designed for the automotive industry, and also become a popular bus in industrial automation as well as other applications in various industries.

The CAN bus is primarily used in embedded systems, and is the network established among microcontrollers. It can be accessed as two-wire, half duplex, high-speed network system.

It is appropriate for high speed applications using short messages. The benefits of CAN such as robustness, reliability made it efficient in industry standard application.

The CAN controller can theoretically link up to 2032 devices on a single network. However, with some of the practical limitation of the hardware (transceivers), but it can only link up to110 nodes (Philips controller) on a single network. It offers top speed communication rate up to $1 \mathrm{Mbits} / \mathrm{sec}$ thus allows real-time control.

The transmission data over the CAN communication line needs to be processed using the CAN controller (MCP 2515) that can be transmitted and received via CAN Transceiver (MCP 2551) along a twisted pair cable. The maximum bus length for a CAN 
network depends on the bit rate used. It is necessary that the wave front of the bit signal has time to travel to the most remote node and back again before the bit is sampled.

\section{H ou se 1}

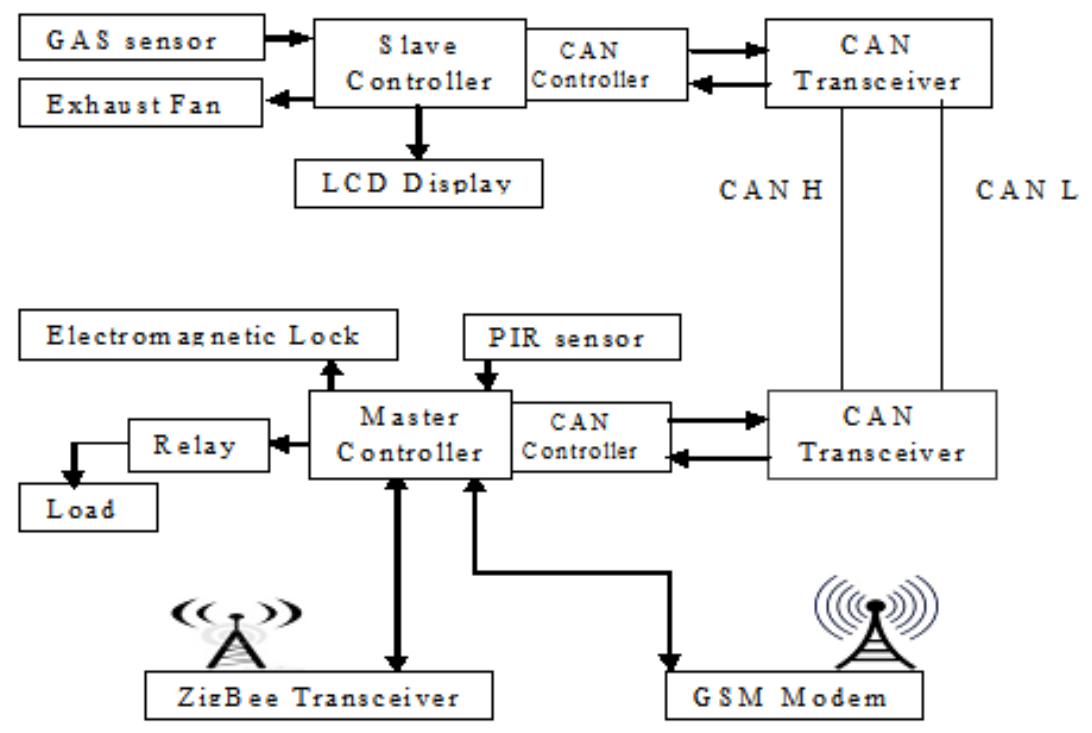

House ow ner \& Police Station

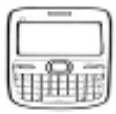

Cen tralized Service Station
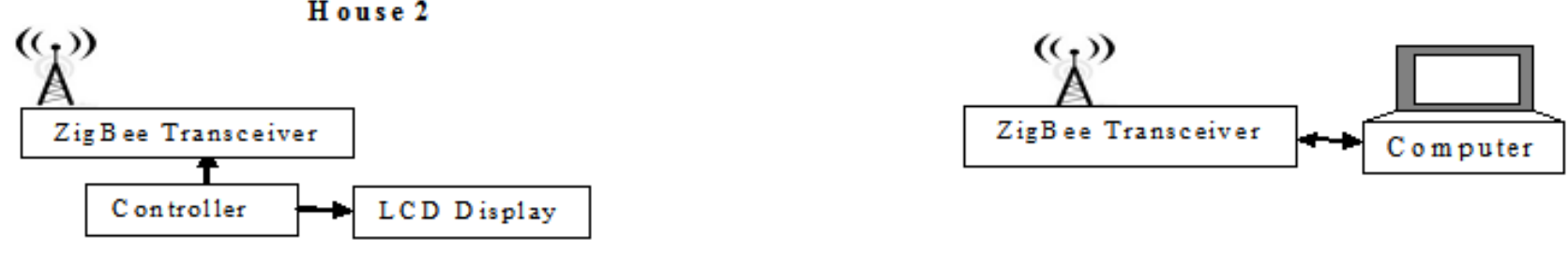

Fig.1. Block Diagram

CAN standards: version 2.0 divided into two parts as given by,

- Standard CAN (Version 2.0A). Uses 11 bit identifiers.

- $\quad$ Extended CAN (Version 2.0B). Uses 29 bit identifiers.

CAN transmits signals on the CAN bus which consists of two wires, a CAN-High and CAN-Low. These 2 wires can operate in differential mode that is they are carrying inverted voltages to decrease noise interference during transmission.

\subsection{PIR Sensor}

The Passive Infrared sensor is used to measure infrared light radiating from an object in its field of it 120 degree view. They are Passive sensors, since they do not have an in built IR source like active sensors and detect the natural radiation of the object.

All objects have a certain body temperature in that specific temperature there is a certain radiation that the body emits called the Black Body Radiation phenomenon. This infrared radiation is undetectable to the human eye but can be detected by this PIR sensor. This PIR output use for alerting the authorized person in the case of detection of human movement inside the house when owner not present in the house.

\subsection{LPG Sensor}

MQ-6 LPG Gas Sensor used in this project can detect up to the range of 200 to $10,000 \mathrm{ppm}$.

When in the state of owner is not present in the house, the gas leakage detection by the system will perform a precautionary function of running the exhaust fan for particular time period. And the system will alert the authority by sending an intimation message. 


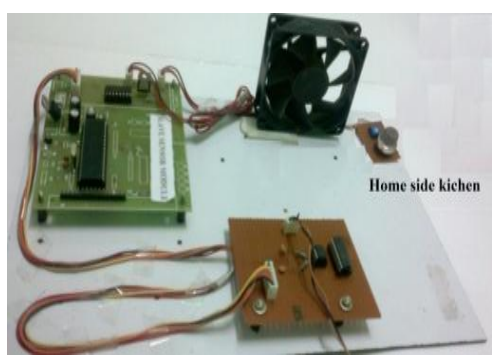

Fig.2. Hardware of Home Kitchen part

\subsection{GSM Modem:}

A GSM modem is a wireless modem that works with a GSM wireless network which can send and receive data through radio waves. The modem uses AT commands instructions used which are used to control a modem, in which AT is the abbreviation of ATtention.

In this project the GSM modem interfaced with master controller that has sensor nodes connected to it, for the purpose of sending alert message to authorized personnel who can take appropriate action for the alert message. And the Message authentication to Open and Close the Electromagnetic Door of the house, Provides more security to the owner and with an enhanced feature of Access permission to all the family members. In other cases the system should send the mobile number of the unauthorized access to owner.

\subsection{Electromagnetic Lock}

Electromagnetic lock is a locking device that consists of an electromagnet and an armature plate. The principle behind an electromagnetic lock is the use of electromagnetism to lock a door when energized. Essentially it consists of an electromagnet attracting a conductor with a force large enough to prevent the door from being opened.

That is to say, if only we could cut off power supply and make electromagnet force between electromagnet and armature plate to disappear, the door would be opened. Furthermore, we just try to use a direct dc output momentary remote and one button transmitter to cut off electric power to open the door. The
Electromagnet Lock can only be accessed through a password message using the GSM modem, and the Probability of network failure/error may be concern for the real time implementation, to eliminate that the Zigbee based remote can be used to open and close the Doors.

\subsection{ZIGBEE Transceiver:}

ZigBee is a wireless technology has been developed as a global standard to address the distinctive needs of low-cost, lowpower wireless Node to node networks. It operates on the physical radio specification of IEEE 802.15.4 standard and operates on $2.4 \mathrm{GHz}, 900 \mathrm{MHz}$ and $868 \mathrm{MHz}$ frequency bands, intended for low-cost, battery-operated devices. This protocol permits devices to communicate in a variety of network topologies and can have battery life for several years.

A key element of the ZigBee protocol is the ability to maintain mesh networking among them. In a mesh network, nodes are interconnected in multiple pathways with each node. The mesh topology allows the nodes to reconfigure routing paths based on the new network structure. The uniqueness of mesh topology and ad-hoc routing provide greater stability in changing conditions or failure of a single node.

\section{CONCLUSIONS AND FUTURE WORK}

The proposed system of wired sensor network based home security system to detect the theft or leaking of raw gas or fire has been developed successfully by implementing CAN bus communication along with the password protected Electromagnetic lock. Inclusion of a ZigBee transceiver in the system, to create a network of ZigBee based houses and to make the system, more secure by sharing security alert messages that could avoid the thievery. This system experienced to be more secure and reliable in real time environment.

The future work of this system will include a centralised control for the group of individual house for immediate response for any kind of alert generated by the individual home.

\subsection{Hardware Interfacing:}

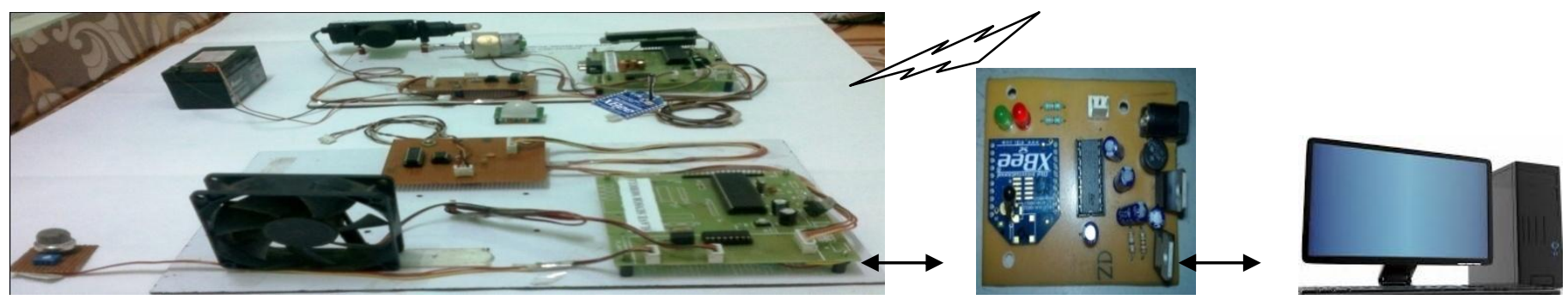

Fig.3. Interfacing circuit of 2 PIC 16F877A using CAN Bus Communication and Zigbee Module Connection with PC. 


\subsection{Mobile Screen Shots}

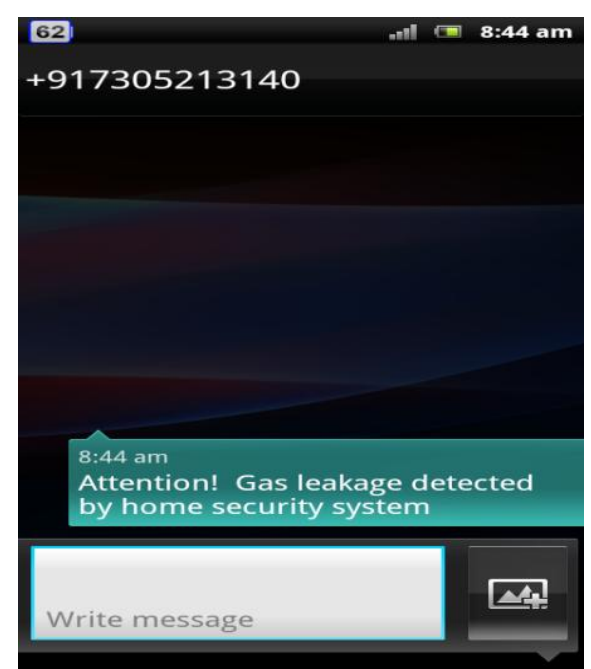

Fig.4. Alert Messages (i) GAS Sensor Alert (ii) PIR Sensor Alert

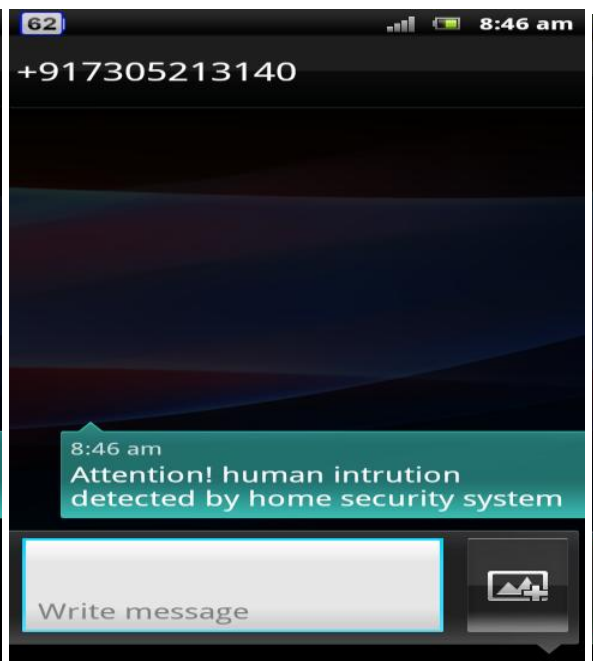

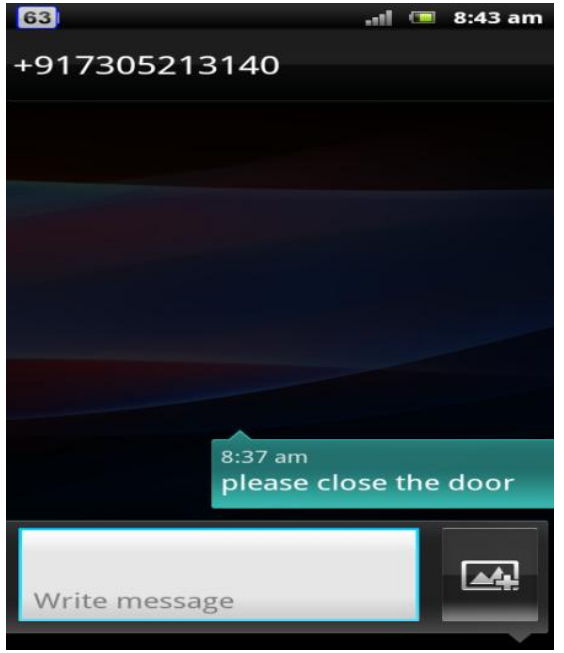

(iii) Warning Message

\section{REFERENCES}

[1] Khusvinder Gill, Shuang-Hua Yang, Wan-Liang Wang, Secure Remote Access To Home Automation Networks, IET Inf. Secur., 2013, Vol. 7, Iss. 2, pp. $118-125$

[2] NausheenBelim, HarshadaBhambure, PriyankaKumbhar, SimranjitTuteja, Automate and Secure Your Home Using Zigbee Technology, The International Journal of Innovative Research in Computer and Communication Engineering, VOL. 1, ISSUE 1, march 2013.

[3] MazranEsro, Amat Amir Basari , Siva Kumar S, A. Sadhiqin M I , ZulkifliSyariff, Controller Area Network Application Insecurity System, World Academy of Science, Engineering and Technology 35 2009.

[4] Dr.S.S.Riaz Ahamed, The Role Of Zigbee Technology In Future Data Communication System, Journal of Theoretical and Applied Information Technology, 2005 $-2009$.

[5] William Stalling, "Wireless Communication and Networks", Fourth Edition, Pearson Publication Limited, 2004, Pp 39-118.

[6] Richards, P. (2005). AN754: Understanding Microchip's CAN Module Bit Timing. Available: http://

www.microchip.com/downloads/en/AppNotes/00754.p df

[7] Al-Ali, A.R., Al-Rousan, M.: 'Java-based home automation system', IEEE Trans. Consum. Electron., 2004, 50, (2), pp. 498-504.

\section{BIOGRAPHIES}

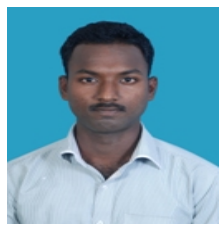

P. Mohan is currently pursuing his Master's degree program in Embedded System Technologies in Rajiv Gandhi College of Engineering, Chennai. He studied his UG degree in Electronics and Communication Engineering Discipline, and his research area includes communication protocols in embedded system.

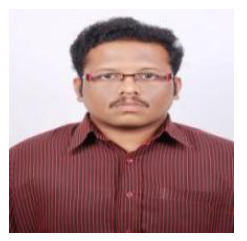

M.Vinoth Kumar is presently working as an Assistant Professor in EEE Dept of Rajiv Gandhi College of Engineering, Chennai. Who have Received M.Tech in Embedded Systems from Hindustan University, Chennai. B.E in ECE from Sengunthar Engineering College, Anna University, Chennai. His research interest includes Mobile Communications, Computer Networks, Embedded Systems and communication networks. . 